\title{
A critical role for extracellular protein disulfide isomerase during thrombus formation in mice
}

\author{
Jaehyung Cho, ${ }^{1}$ Barbara C. Furie, ${ }^{1}$ Shaun R. Coughlin, ${ }^{2}$ and Bruce Furie ${ }^{1}$ \\ 1Division of Hemostasis and Thrombosis, Beth Israel Deaconess Medical Center and Harvard Medical School, Boston, Massachusetts, USA. \\ ${ }^{2}$ Cardiovascular Research Institute, University of California San Francisco, San Francisco, California, USA.
}

\begin{abstract}
Thiol isomerases, including protein disulfide isomerase (PDI), catalyze disulfide oxidation, reduction, and isomerization, thereby playing an important role in protein synthesis. To determine whether extracellular PDI mediates thrombus formation in an animal model, PDI expression, platelet accumulation, and fibrin generation were monitored in the blood vessels of mice by intravital fluorescence microscopy following laser-induced arteriolar injury. A time-dependent increase in PDI was observed in murine thrombi following injury. Infusion of the PDI inhibitor bacitracin or a blocking monoclonal antibody against PDI inhibited platelet thrombus formation and fibrin generation. Fibrin deposition is normal in mice lacking the $G$ protein-coupled platelet receptor Par4, although there is no stable accumulation of platelets. Infusion of monoclonal antibodies against PDI into the circulation of $\mathrm{Par}^{-/-}$mice prior to vessel wall injury inhibited fibrin generation. These results indicate that PDI is required in vivo in mice for both fibrin generation and platelet thrombus formation.
\end{abstract}

\section{Introduction}

Evidence is mounting that important steps in the process of thrombus formation may be regulated by the oxidation states of labile disulfide bonds in critical hemostatic proteins (1). The formation of disulfide bonds is catalyzed by protein disulfide isomerase (PDI) or other PDI family member thiol isomerases. All of these family members are found in the endoplasmic reticulum, where they play important roles during protein synthesis. In spite of having endoplasmic reticulum retention signals, PDI and other thiol isomerases have been identified in cellular locations outside of the endoplasmic reticulum. Platelets are among the cells that secrete and display PDI on their surface $(2,3)$. Reduced glutathione and cysteine impact platelet aggregation $(4,5)$; this suggests a role for thiol/disulfide exchange in the aggregation process. Thiol isomerases likely play an important role in maintaining this balance.

PDI has been implicated in the alteration of $\alpha_{\mathrm{IIb}} \beta_{3}$ and $\alpha_{2} \beta_{1}$ activities $(6,7)$. Inhibitory anti-PDI antibodies or bacitracin, a nonspecific inhibitor of thiol isomerases, inhibit platelet activation in vitro, suggesting that $\alpha_{\mathrm{IIb}} \beta_{3}$-dependent platelet aggregation and secretion require thiol isomerases. Glycoprotein Ibo expresses 1 or more free thiols on the activated platelet surface, but not on resting platelets (8). Furthermore, PDI may participate in the conversion of encrypted tissue factor to its active form in a favorable oxidative environment $(9,10)$.

The work of Hogg has led to a novel proposal that the oxidation state of surface-exposed labile disulfide bonds, -RHStaples, can be regulated to alter protein function (11). A survey of the Protein Data Bank identified only 118 functionally distinct proteins with 1 or more-RHStaple bonds $(12,13)$. Among these, proteins important to hemostasis and thrombosis include tissue factor, thrombospondin-1, plasmin, von Willebrand factor, and the $\beta_{3}$ integrin subunit. During their extracellular lifetimes, these proteins may undergo either cleavage or formation of disulfide bonds, which have significant implications for the proteins' structure and function.

Nonstandard abbreviations used: PDI, protein disulfide isomerase. Conflict of interest: The authors have declared that no conflict of interest exists. Citation for this article: J. Clin. Invest. 118:1123-1131 (2008). doi:10.1172/JCI34134.
To understand the physiologic role of extracellular PDI during thrombus formation, we studied PDI expression, platelet accumulation, and fibrin deposition in a mouse thrombosis model using laser injury of the arteriole vessel wall. In vivo studies monitoring the expression of PDI demonstrated the rapid accumulation of PDI in the developing thrombus following injury. Thrombus formation - both platelet accumulation and fibrin deposition - was completely inhibited in vivo when either bacitracin or an inhibitory anti-PDI antibody was infused prior to vessel wall injury. These results indicate a critical requirement of PDI in thrombus formation in vivo.

\section{Results}

We have developed a system for imaging and analyzing thrombus formation in a live mouse using laser-induced injury. Platelet activation in this model is dominated by the initiation of the tissue factor pathway that leads to thrombin generation $(14,15)$. In response to the recent proposals that tissue factor can be stabilized by PDI in a form characterized by low coagulant activity that maintains signaling activity $(9,16)$ and that PDI stimulates tissue factor independent of its oxidoreductase activity (10), we used this in vivo model to ascertain the physiologic role of PDI in thrombus development in an animal model.

Expression of PDI during thrombus formation in vivo. We used intravital microscopy to image the expression of PDI in the developing thrombus after laser injury in a mouse cremaster muscle arteriole. PDI was visualized using IgG isolated from polyclonal anti-bovine PDI antiserum. This IgG fraction was either directly labeled with Alexa Fluor 488 or immunoaffinity purified on a PDI-agarose column and then labeled with Alexa Fluor 488. By Western blot, we observed both of these rabbit anti-bovine PDI antibody preparations to be reactive with both human and mouse PDI, with PDI appearing as the major band in an immunoblot of platelet lysate (Figure 1A). In contrast, IgG isolated from nonimmune antiserum and labeled with Alexa Fluor 488 showed no reactivity to PDI. At the concentrations used for imaging, this antibody did not inhibit PDI activity or perturb thrombus formation.

No PDI was observed on the unperturbed vessel wall. PDI accumulated rapidly in the thrombus that evolved at the site of laser 


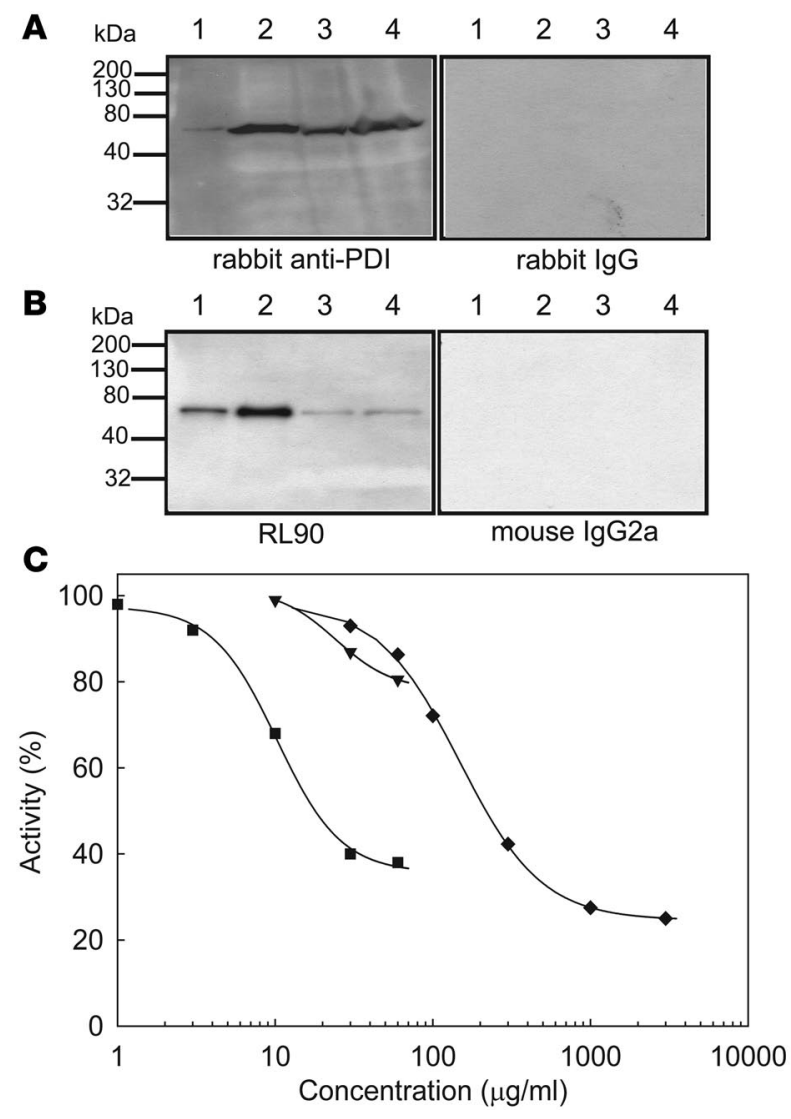

injury (Figure 2A, left). PDI antigen expression increased in a time-dependent manner and was observed within $15 \mathrm{~s}$ of vessel wall injury. Minimal fluorescence was detected with infusion of Alexa Fluor 488-labeled nonimmune rabbit IgG (Figure 2A, right). Quantitative data were obtained by analysis of multiple thrombi. The time course of the median integrated fluorescence associated with PDI for 32 thrombi generated in 3 mice showed that the PDI accumulated early during thrombus formation, with peak fluorescence at about 50-80 s (Figure 2B). In control experiments on 28 thrombi in 3 mice, no increase in fluorescence associated with nonimmune IgG conjugated to Alexa Fluor 488 was detected over time. These results suggest that PDI is rapidly expressed within the developing thrombus following vessel wall injury and remains associated with the thrombus.

PDI expression precedes platelet accumulation. To compare the kinetics of PDI expression with those of platelet accumulation, PDI and platelets were monitored simultaneously using affinitypurified anti-PDI antibodies conjugated to Alexa Fluor 488 and a Fab fragment of the anti-CD41 antibody conjugated to Alexa Fluor 647 following laser-induced injury of the vessel wall. PDI appeared within $15 \mathrm{~s}$ of vessel wall injury, whereas initial platelet accumulation was observed at about $30 \mathrm{~s}$ (Figure 3A, left). When nonimmune IgG and platelets were monitored simultaneously using nonimmune IgG conjugated to Alexa Fluor 488 and a Fab fragment of the anti-CD41 antibody conjugated to Alexa Fluor 647 following laser-induced injury of the vessel wall, only platelets were detected in the developing thrombus (Figure 3A, right). The median integrated fluorescence associated with platelet accumulation for 28 thrombi generated in 3 mice indicated early accumula-

\section{Figure 1}

Interaction of anti-PDI antibodies with human and mouse PDI. Lysates of human $\left(1 \times 10^{9}\right.$ platelets $\left./ \mathrm{ml}\right)$ and mouse $\left(1 \times 10^{10}\right.$ platelets $\left./ \mathrm{ml}\right)$ platelets were subjected to SDS gel electrophoresis followed by immunoblotting with anti-PDI antibodies. (A) Polyclonal anti-PDI antibodies. Lane 1, recombinant human PDI (20 ng); lane 2, human platelet lysate $(20 \mu \mathrm{l})$; lane 3 , mouse platelet lysate $(5 \mu \mathrm{l})$; lane 4 , mouse platelet lysate $(20 \mu \mathrm{l})$. Left: Immunoblot developed using affinity-purified rabbit anti-bovine PDI antibody. Right: Immunoblot developed with control nonimmune IgG. (B) Monoclonal antibody RL90 to PDI. Lane 1, recombinant human PDI (1 ng); lane 2, human platelet lysate $(1 \mu \mathrm{l})$; lane 3 , mouse platelet lysate $(5 \mu \mathrm{l})$; lane 4 , mouse platelet lysate $(20 \mu \mathrm{l})$. Left: Immunoblot developed using the RL90 anti-PDI monoclonal antibody. Right: Immunoblot developed with control IgG2a monoclonal antibody. (C) PDI activity was measured with the insulin transhydrogenase assay in the releasate of thrombin-activated mouse platelets $(n=3)$ in the presence or absence of $1-60 \mu \mathrm{g} / \mathrm{ml} \mathrm{RL90} \mathrm{mono-}$ clonal anti-PDI antibody (squares), $10-60 \mu \mathrm{g} / \mathrm{ml}$ rabbit anti-PDI antibody (inverted triangles), or $0.03-3.0 \mathrm{mg} / \mathrm{ml}$ bacitracin A (diamonds). Results are shown as a mean of 2 experiments.

tion just prior to $50 \mathrm{~s}$ and peak accumulation at about $90-100 \mathrm{~s}$ (Figure 3B). The median integrated fluorescence associated with PDI for these same thrombi showed the early appearance of fluorescence associated with PDI at about $10 \mathrm{~s}$, with peak fluorescence at about 70-90 s (Figure 3C). These results indicate that initial PDI expression occurs prior to significant platelet accumulation. The source of the PDI detected is unclear: the initial PDI may derive from endothelial cells or may be contributed from other cells, and later accumulation of PDI likely derives from platelets, although endothelial cells or other cells may contribute.

To further assess the specificity of the affinity-purified polyclonal anti-PDI antibody in vivo, exogenous bovine PDI was infused into the mice as a competitor for PDI appearing at the site of thrombus formation. After observing labeling of both platelets and PDI accumulation in 6 separate thrombi as described above, exogenous bovine PDI $(100 \mu \mathrm{g})$ was infused through the jugular catheter, and 6 additional thrombi were generated. In the presence of circulating exogenous bovine PDI, no fluorescently labeled anti-PDI antibody accumulation was detected in the thrombus, although platelet accumulation was not altered (Supplemental Figure 1; available online with this article; doi:10.1172/JCI34134DS1). These experiments showed that the affinity-purified anti-PDI antibodies that accumulate in vivo are indeed antibodies that recognize PDI.

A thiol isomerase inhibitor blocks platelet thrombus formation and fibrin generation. Bacitracin is an inhibitor of PDI and other thiol isomerases in the PDI family. To determine the effect of thiol isomerase inhibition on fibrin deposition and platelet thrombus formation following laser injury, bacitracin was infused into the mouse circulation 5 min prior to vessel wall injury, and fibrin deposition and platelet accumulation were imaged simultaneously. Platelet accumulation was monitored in the developing thrombus using Alexa Fluor 647-labeled Fab fragments of CD41 antibodies, and fibrin deposition was monitored using an Alexa Fluor 488-labeled fibrinspecific antibody that is not reactive with fibrinogen. Increasing concentrations of bacitracin led to decreased fibrin deposition and platelet accumulation after vessel wall injury (Figure 4A). Quantitative data were obtained by analysis of multiple thrombi. The median integrated platelet fluorescence and the median integrated fibrin fluorescence presented as a function of time for thrombi formed following infusion of $0,0.3,1.5$, and $5.0 \mathrm{mg}$ of bacitracin per mouse 
A
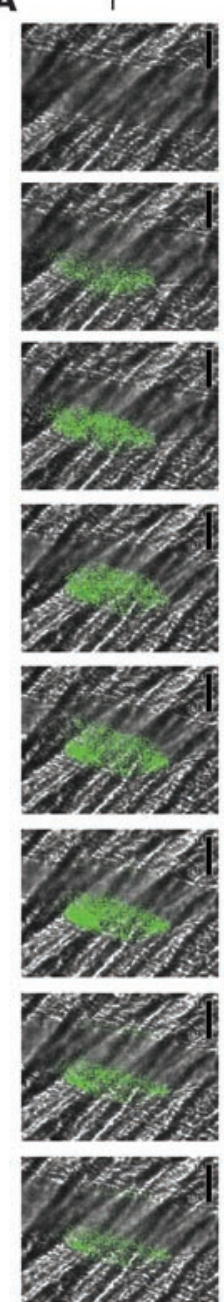

2

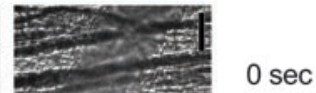

$0 \mathrm{sec}$
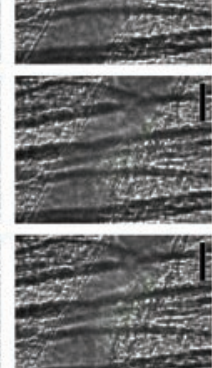

$30 \mathrm{sec}$
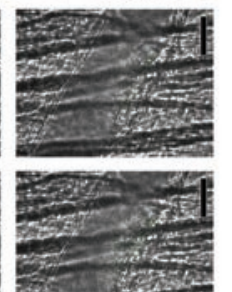

$90 \mathrm{sec}$

B

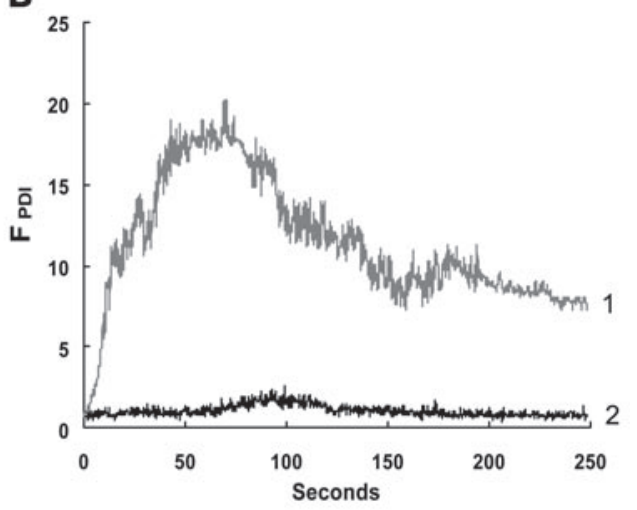

Figure 2

Expression of PDI during thrombus formation in vivo. A nonblocking polyclonal anti-PDI antibody conjugated to Alexa Fluor 488 or an irrelevant IgG conjugated to Alexa Fluor $488(0.3 \mu \mathrm{g} / \mathrm{g} \mathrm{BW})$ was infused into a mouse 5 min prior to arteriolar injury. (A) Time course of appearance of the anti-PDI antibody (column 1) or the irrelevant IgG (column 2) over $240 \mathrm{~s}$ following vessel wall injury. (B) Median integrated PDI fluorescence for 32 thrombi in 3 wild-type mice infused with the anti-PDI antibody (curve 1) and for 28 thrombi in 3 wildtype mice infused with the irrelevant IgG (curve 2) are presented versus time after vessel wall injury. A representative binarized image is shown in A, and the complete data sets of this and multiple identical experiments are presented in $\mathbf{B}$, which plots the integrated fluorescence intensity of all pixels in the image, regardless of their intensity, as a function of time. indicated a dose-dependent inhibition of platelet accumulation (Figure 4B) and fibrin deposition (Figure 4C) that were approximately parallel. At the 5.0-mg bacitracin dose, no fibrin was visualized. Thiol isomerases have been implicated in posttranslational modification of multiple components that are important in thrombus formation, including $\alpha_{\mathrm{II}} \beta_{3}$ and tissue factor (1). The inhibition of platelet accumulation and fibrin deposition by bacitracin may be the result of inhibited thiol isomerase-mediated modification of these components or others involved in thrombus formation.

Inbibitory PDI antibody blocks platelet thrombus formation and fibrin generation. To distinguish the effect of PDI from that of other thiol isomerases on thrombus formation, fibrin deposition and platelet accumulation were monitored simultaneously in the presence of an inhibitory monoclonal antibody to PDI. This antibody, RL90, bound to and inhibited PDI but did not interact with other thiol isomerases in platelet (Figure 1, B and C) or endothelial cell releasates (R. Jasuja, B.C. Furie, and B. Furie, unpublished observations). Fibrin deposition and platelet accumulation were detected as described above. Increasing concentrations of anti-PDI antibody infused into the circulation prior to vessel wall injury led to decreasing fibrin deposition and platelet accumulation (Figure 5A). Quantitative data were obtained by analysis of multiple thrombi. The median integrated fluorescence associated with platelets and the median integrated fluorescence associated with fibrin presented as a function of time for thrombi formed following infusion of $0,0.3$, 1.0, and $3.0 \mu \mathrm{g} / \mathrm{g}$ BW RL90 indicated a dose-dependent inhibition of platelet accumulation (Figure 5B) and fibrin deposition (Figure 5C). At the 3.0- $\mu \mathrm{g} / \mathrm{g} \mathrm{BW}$ dose of anti-PDI antibody, no fibrin was visualized, and minimal platelet accumulation was observed. These results suggest that the thiol isomerase targets involved in inhibition of thrombus formation by bacitracin under the current experimental conditions include PDI. Moreover, PDI appears to play a necessary role in both platelet accumulation and fibrin deposition during thrombus formation in this laser injury model because these processes were blocked when PDI was inhibited. Although other thiol isomerases may have important functions during thrombosis, their effect may be distal to the steps where PDI is required.

Fibrin generation in the Par4 ${ }^{-/}$mouse. PDI is known to interact both with tissue factor and with platelet surface proteins $(8,9,17)$. To determine whether PDI affects platelet accumulation independent of its effects on coagulation and whether it inhibits fibrin formation independent of its effects on platelets, we explored fibrin deposition in the Par4 $4^{-/}$mouse. Platelets from $\mathrm{Par}^{4^{--}}$mice fail to respond to thrombin (18). Although there is initial platelet accumulation following laser-induced arteriolar injury in these mice, platelet accumulation remains minimal (19). Using P-selectin as 
A
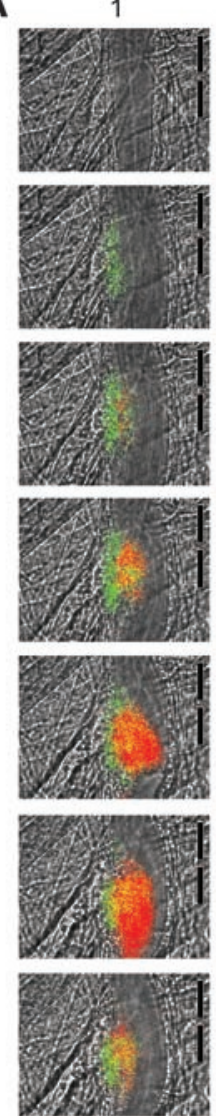

2

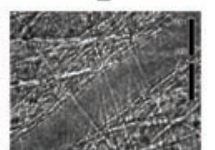

$0 \mathrm{sec}$

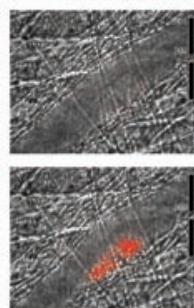

$15 \mathrm{sec}$
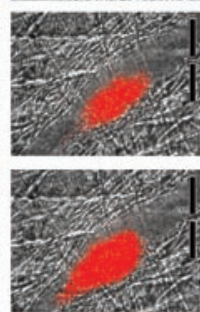

$60 \mathrm{sec}$

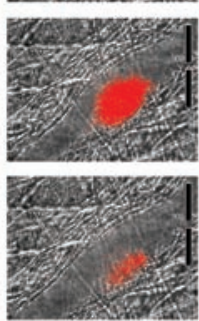

B

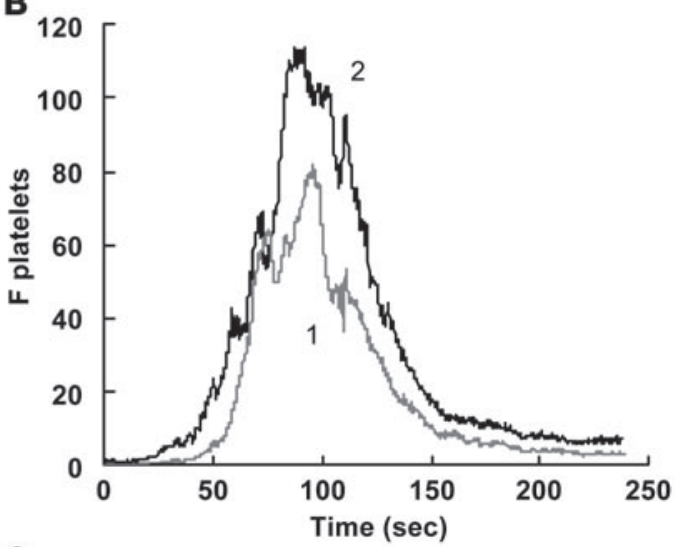

C

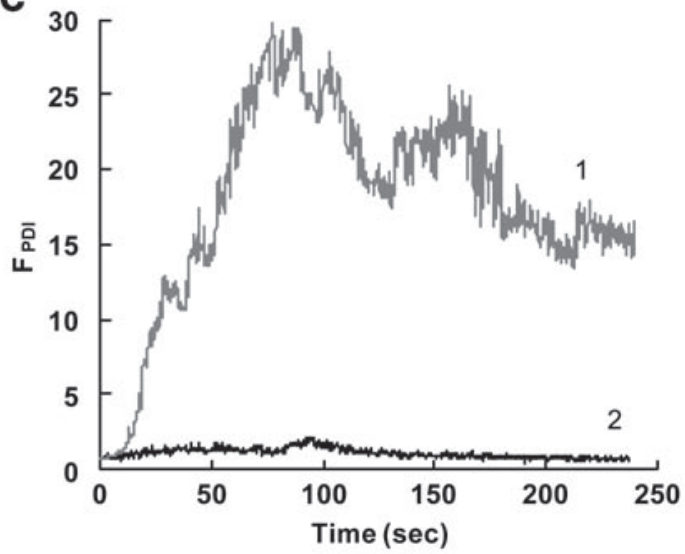

Figure 3

Comparison of PDI expression and platelet accumulation. Rabbit polyclonal anti-PDI antibodies conjugated to Alexa Fluor $488(0.3 \mu \mathrm{g} / \mathrm{g}$ BW) and Fab fragments of anti-CD41 antibodies conjugated to Alexa Fluor $647(0.3 \mu \mathrm{g} / \mathrm{g} \mathrm{BW})$ were infused into a mouse 5 min prior to arteriolar injury. (A) Time course of appearance of the fluorescence signals associated with PDI (green) and platelets (red) over $180 \mathrm{~s}$ following laser-induced vessel wall injury in wild-type mice within the context of the bright-field microvascular histology (column 1). In control experiments, anti-PDI antibodies were replaced with nonimmune IgG conjugated to Alexa Fluor 488 (column 2). (B and C) Median integrated platelet fluorescence (B) and median integrated PDI fluorescence (C) after infusion of rabbit affinity-purified anti-PDI antibodies (curve 1; 28 thrombi, 3 wild-type mice) or rabbit nonimmune IgG (curve 2; 32 thrombi, 3 wild-type mice) are presented versus time after vessel wall injury. A representative binarized image is shown in $\mathbf{A}$, and the complete data sets of this and multiple identical experiments are presented in $\mathbf{B}$ and $\mathbf{C}$, which plot the integrated fluorescence intensity of all pixels in the image, regardless of their intensity, as a function of time.

a marker for platelet activation, we have previously demonstrated in an in vivo observation that the platelets in the juxtamural thrombus that forms in Par4 $4^{-/}$mice are activated in the laser injury model only after a long delay, suggesting that platelets within the juxtamural thrombi can not support fibrin generation (19). However, fibrin deposition in Par4 $4^{-/}$mice is equivalent to that in wild-type mice after laser injury, suggesting that another membrane surface is responsible for supporting fibrin generation (19). Platelets and fibrin were simultaneously imaged following vessel wall injury in $\mathrm{Par}^{-/-}$mice in the presence or absence of the inhibitory anti-PDI antibody RL90. Inhibition of PDI eliminated fibrin accumulation in Par4 ${ }^{-/}$mice (Figure 6, A and C), strongly suggesting that PDI plays a necessary role in thrombin generation in this model. Inhibition of PDI also eliminated the small juxtamural thrombus usually formed in $\mathrm{Par}^{-/-}$mice (Figure 6, A and B). Because the juxtamural platelet thrombus in Par4 ${ }^{-/}$mice is independent of platelet activation by thrombin, these results are consistent with an effect of PDI on platelet function that is independent of its impact on thrombin generation.
Effect of antibodies to PDI on tail bleeding time. We performed analyses of tail bleeding times in mice treated with the anti-PDI monoclonal antibody RL90 or an isotype-matched control using 2 separate end points. Mice were treated with $0.3,1.0$, or $3 \mu \mathrm{g} / \mathrm{g}$ BW of RL90 or with $3 \mu \mathrm{g} / \mathrm{g} \mathrm{BW}$ of an irrelevant IgG2a monoclonal antibody. Bleeding was measured by the cessation of blood loss (Figure 7A) and by quantitation of hemoglobin loss (Figure 7B). In the mice treated with $3 \mu \mathrm{g} / \mathrm{g}$ BW RL90, the median tail bleeding time was significantly longer and the median hemoglobin loss significantly greater than in the control mice treated with $3 \mu \mathrm{g} / \mathrm{g} \mathrm{BW}$ of a nonimmune IgG2a antibody. While we observed inhibition of thrombus formation in vivo at the 2 lower doses of RL90 used in the tail bleeding experiment, complete inhibition of thrombus formation in vivo was only observed at the $3.0-\mu \mathrm{g} / \mathrm{g} \mathrm{BW}$ dose (Figure 5).

\section{Discussion}

We used an in vivo thrombosis model to evaluate the role of thiol isomerases in thrombus formation in vivo. In a laser injury model, we found that PDI antigen was detected before the onset of plate- 
A
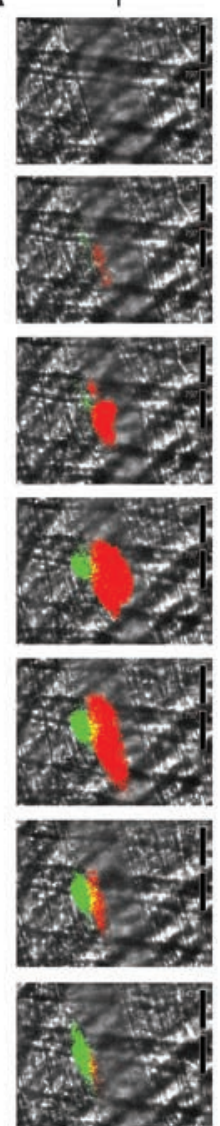

2
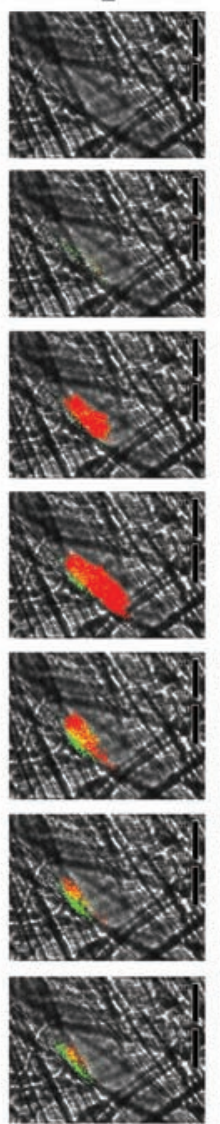

3
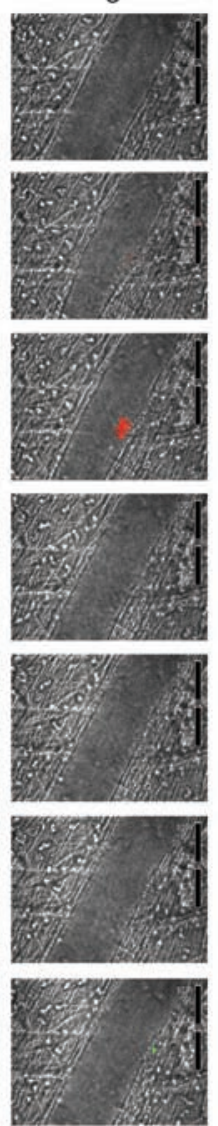

4
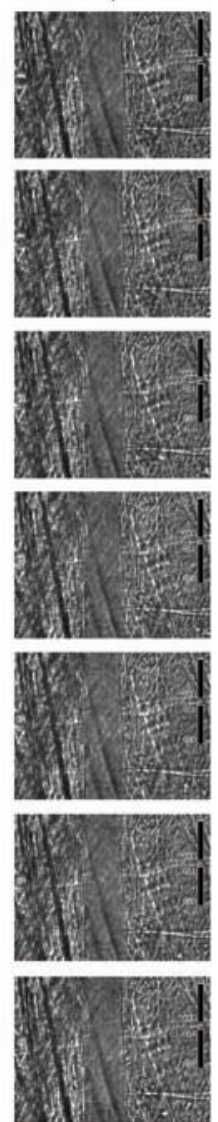

B

$0 \mathrm{sec}$

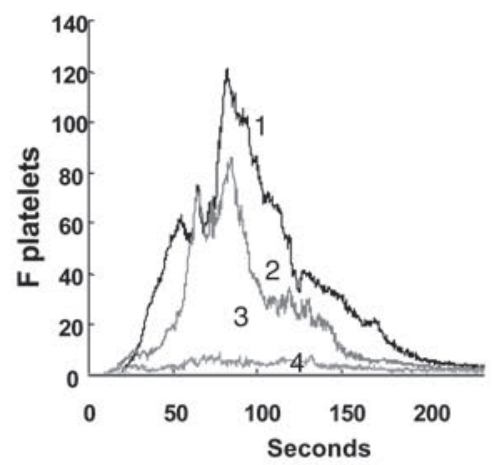

$60 \mathrm{sec}$

$90 \mathrm{sec}$

C

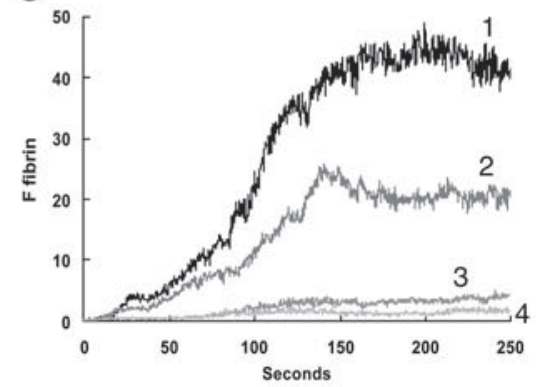

Figure 4

Inhibition of fibrin formation and platelet accumulation in wild-type mice as a function of increasing concentrations of bacitracin A. (A) Fibrin was labeled with a fibrin-specific antibody conjugated to Alexa Fluor 488, and platelets were labeled with Fab fragments of anti-CD41 antibodies conjugated to Alexa Fluor 647. The time course of appearance of fluorescence associated with fibrin (green) and platelets (red) is shown over $180 \mathrm{~s}$ following laser-induced vessel wall injury in wild-type mice within the context of the bright-field microvascular histology. Bacitracin A was infused into the circulation $5 \mathrm{~min}$ prior to injury at $0,0.3,1.5$, and $5.0 \mathrm{mg}$ per mouse (columns $1-4$, respectively). (B and C) Median integrated platelet fluorescence (B) and median integrated fibrin fluorescence $(\mathbf{C})$ for thrombi formed in the presence of increasing doses of bacitracin $\mathrm{A}-0 \mathrm{mg}$ (curve 1; 24 thrombi, 3 mice), $0.3 \mathrm{mg}$ (curve 2; 27 thrombi, 3 mice), $1.5 \mathrm{mg}$ (curve 3; 22 thrombi, 3 mice), and $5.0 \mathrm{mg}$ (curve 4; 20 thrombi, 3 mice) - are presented versus time after vessel wall injury. A representative binarized image is shown in $\mathbf{A}$, and the complete data sets of this and multiple identical experiments are presented in $\mathbf{B}$ and $\mathbf{C}$, which plot the integrated fluorescence intensity of all pixels in the image, regardless of their intensity, as a function of time.

let and fibrin accumulation. Moreover, infusion of 2 structurally dissimilar PDI inhibitors blocked both platelet and fibrin accumulation in this model.

The source of the PDI that appears during thrombus formation remains to be determined. Because PDI has not been detected in plasma by others (20) or ourselves (our unpublished observations), the PDI visualized during thrombus formation presumably derives from cells that release PDI upon activation or injury. Activated platelets can release catalytically active PDI $(2,20)$; thus, platelet PDI may contribute to the accumulating PDI antigen associated with the thrombus. PDI released from activated or injured endothelial cells or other vessel wall components might also participate.

The ability of PDI inhibitors to abolish fibrin formation strongly suggests that PDI plays a necessary role in local thrombin generation in the laser injury model. Moreover, because fibrin deposition in Par4 -/ mice is independent of platelets or requires minimal plate- let activation or accumulation, the dramatic effect of PDI inhibitors on fibrin accumulation in $\mathrm{Par}^{-/-}$mice suggests that these inhibitors may function by a platelet-independent mechanism.

Tissue factor antigen can be detected at the site of vessel wall injury immediately following laser insult in our model (21). In vitro studies have led to the proposal that PDI may be associated with an encrypted form of tissue factor and may regulate tissue factor activity $(9,16)$. Thus, inhibition of extracellular PDI is a potential mechanism for the platelet-independent inhibition of thrombin generation and fibrin deposition that we observed after laser-induced vessel wall injury in the presence of PDI inhibitors. However, the concept that thiol isomerase-catalyzed oxidation of an allosteric disulfide bond in tissue factor leads to the activation of tissue factor has been controversial for 4 reasons: (a) Bach et al. demonstrated that purified full-length tissue factor has no free thiols in the extracellular domain (22); (b) oxidants such as mercuric chloride that modify tissue factor activity might target a criti- 
A
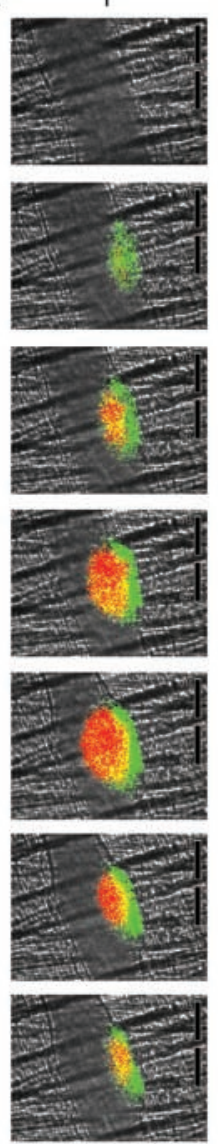
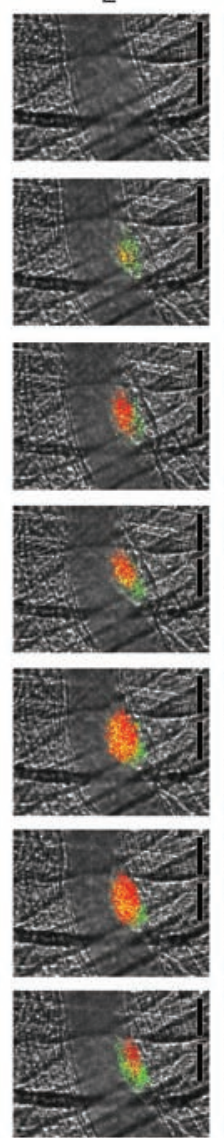
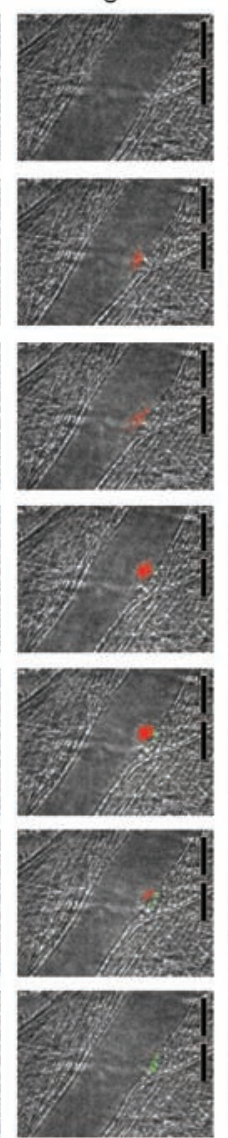

4
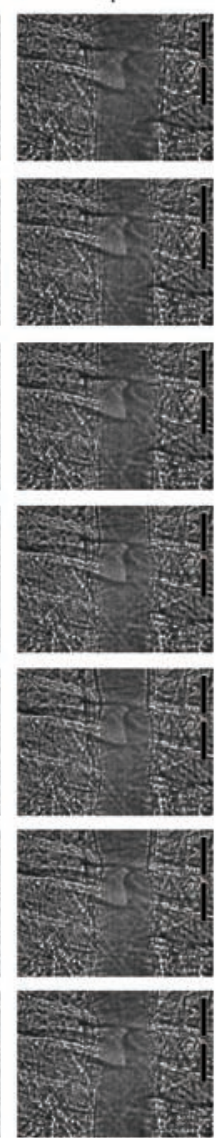

$0 \mathrm{sec}$

$15 \mathrm{sec}$

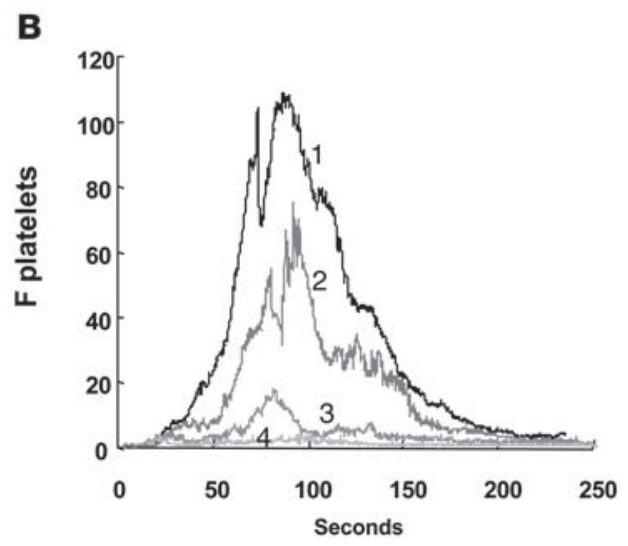

$60 \mathrm{sec}$

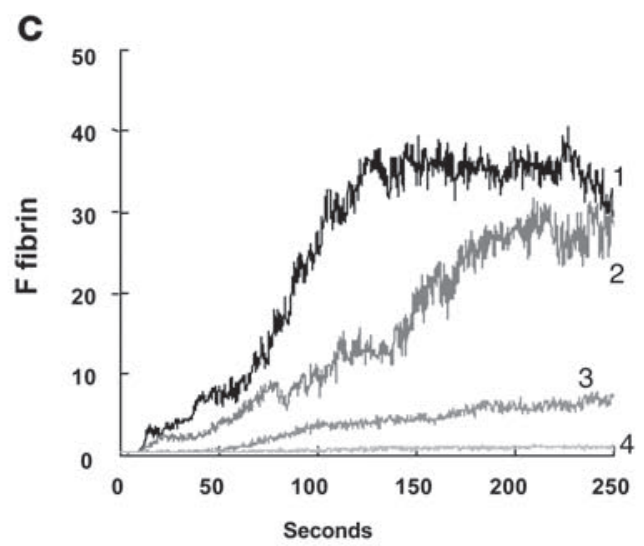

$180 \mathrm{sec}$

\section{Figure 5}

Inhibition of fibrin formation and platelet accumulation in wild-type mice as a function of increasing concentrations of inhibitory RL90 antibody to PDI. (A) Fibrin was labeled with a fibrin-specific antibody conjugated to Alexa Fluor 488, and platelets were labeled with Fab fragments of anti-CD41 antibodies conjugated to Alexa Fluor 647. The time course of appearance of fluorescence associated with fibrin (green) and platelets (red) is shown over $180 \mathrm{~s}$ following laser-induced vessel wall injury in wild-type mice within the context of the bright-field microvascular histology. Inhibitory monoclonal anti-PDI antibody RL90 was infused into the circulation 5 min prior to injury at 0, 0.3, 1.0, and 3.0 $\mu \mathrm{g} / \mathrm{g} \mathrm{BW}$ (columns $1-4$, respectively). In all experiments when no inhibitory monoclonal anti-PDI antibody was infused, $3.0 \mu \mathrm{g} / \mathrm{g}$ BW of isotype-matched IgG2a was infused instead. (B and C) Median integrated platelet fluorescence (B) and median integrated fibrin fluorescence (C) for thrombi formed in the presence of increasing concentrations of the anti-PDI antibody RL90 - $0 \mu \mathrm{g}$ (curve 1; 27 thrombi, 3 mice), $0.3 \mu \mathrm{g}$ (curve 2; 28 thrombi, 3 mice), $1.0 \mu \mathrm{g}$ (curve 3; 23 thrombi, 3 mice), and $3.0 \mu \mathrm{g}$ (curve 4; 21 thrombi, 3 mice) - are presented versus time after vessel wall injury. A representative binarized image is shown in $\mathbf{A}$, and the complete data sets of this and multiple identical experiments are presented in $\mathbf{B}$ and $\mathbf{C}$, which plot the integrated fluorescence intensity of all pixels in the image, regardless of their intensity, as a function of time.

cal chemical moiety other than a free thiol on tissue factor (16); (c) PDI might be a required cofactor for tissue factor coagulant activity but not participate as an oxidoreductase (10); and (d) reexamination of this disulfide switching mechanism in tissue factor, albeit with different cells and different experimental conditions, has not confirmed the original report (23). Thus, while PDI might promote coagulation by switching a disulfide bond in tissue factor, we do not exclude other mechanisms and other PDI targets.

The observation in $\mathrm{Par}^{-/-}$mice that PDI inhibitors blocked formation of juxtamural platelet thrombi, whose formation does not require platelet activation by thrombin, suggests that PDI inhibitors may do more than simply reduce thrombin generation. Previous in vitro work has shown that specific platelet receptor activities can be modified by inhibition of PDI. Blocking thiol isomerase activity with inhibitory anti-PDI antibodies, low-molecular weight sulfhydryl reagents, or bacitracin all prevent activation of the fibrinogen receptor $\alpha_{\mathrm{II}} \beta_{3}$ after platelet stimulation with a variety of agonists in vitro $(6,24-26)$. It has been proposed that activation of $\alpha_{\text {II }} \beta_{3}$ involves disulfide rearrangement with the number of free sufhydryls, localized to the disulfide bonded knot between residues 400 and 650 , increasing in the $\beta_{3}$ subunit upon activation (27). This receptor undergoes a conformational alteration during conversion from the low-affinity to the high-affinity form of the integrin. More recently, Cys663-Cys687 was identified as a potential allosteric disulfide bond (1), and mutation of Cys663 and Cys687 to alanine resulted in a constitutively active $\alpha_{\mathrm{II}} \beta_{3}(28)$. How alteration of these bonds might relate to inside-out and outside-in signaling pathways is unknown. Nonetheless, it is possible that the thrombin-independent effects of PDI inhibitors in the laser model relate to altering the function of the fibrinogen receptor.

The observation that PDI accumulated at a site of laser injury and the dramatic effect of acute administration of PDI inhibitors 
A
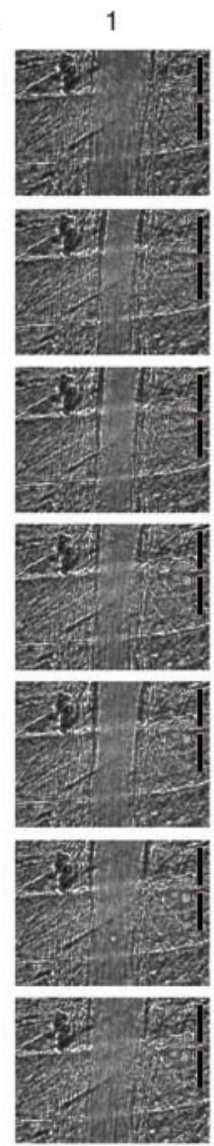

2

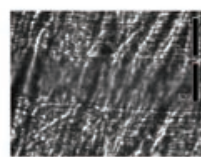

$0 \mathrm{sec}$

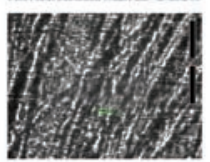

$15 \mathrm{sec}$

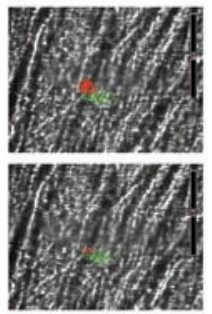

$30 \mathrm{sec}$

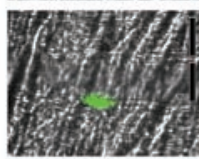

$90 \mathrm{sec}$
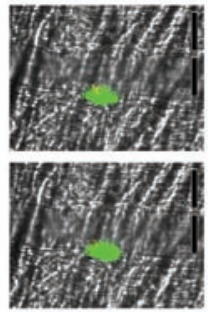

B
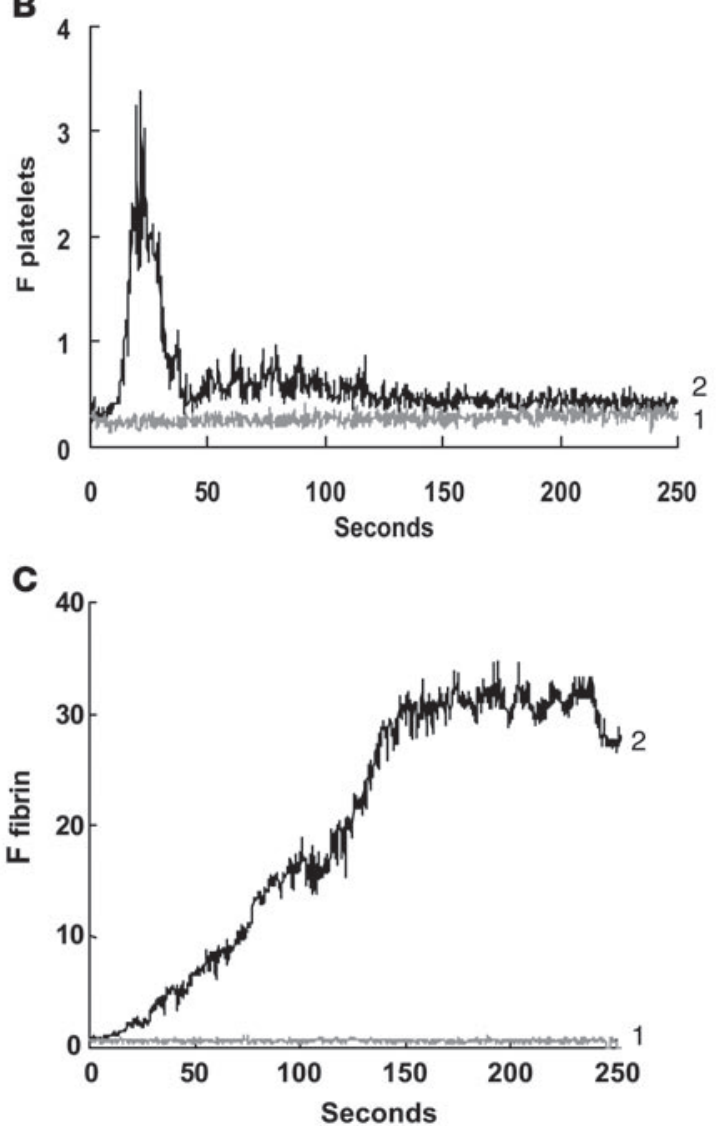

Figure 6

Inhibition of fibrin formation by an inhibitory antibody to PDI in the Par4-/- mouse. (A) Fibrin was labeled with a fibrin-specific antibody conjugated to Alexa Fluor 488, and platelets were labeled with Fab fragments of anti-CD41 antibodies conjugated to Alexa Fluor 647. The time course of appearance of fluorescence associated with fibrin (green) and platelets (red) is shown over $250 \mathrm{~s}$ following laser-induced vessel wall injury in $\mathrm{Par4}^{-/-}$mice within the context of the bright-field microvascular histology. Inhibitory monoclonal anti-PDI antibody RL90 (2 $\mu \mathrm{g} / \mathrm{g}$ BW; column 1) or an irrelevant isotype matched IgG2a antibody ( $2 \mu \mathrm{g} / \mathrm{g} \mathrm{BW}$; column 2) was infused into the circulation 5 min prior to injury. (B and C) Median integrated platelet fluorescence $(\mathbf{B})$ and median integrated fibrin fluorescence $(\mathbf{C})$ for 24 thrombi in 3 Par4 $4^{-/-}$mice infused with the irrelevant IgG2a antibody (curve 1) and for 20 thrombi in 3 Par4 $4^{--}$mice infused with RL90 antibody (curve 2) are presented versus time after vessel wall injury. A representative binarized image is shown in $\mathbf{A}$, and the complete data sets of this and multiple identical experiments are presented in $\mathbf{B}$ and $\mathbf{C}$, which plot the integrated fluorescence intensity of all pixels in the image, regardless of their intensity, as a function of time.

on inhibiting thrombus formation and prolonging tail bleeding time in mice strongly suggest a key role for PDI in fibrin deposition and platelet accumulation during thrombus formation in vivo. Furthermore, PDI represents a unique antithrombotic target for novel pharmaceuticals because its inhibition blocks both platelet thrombus formation and fibrin formation. However, enthusiasm for this approach must be tempered pursuant to careful evaluation in animal models of the possible risk-benefit ratio of such interventions.

\section{Methods}

Mice. Wild-type C57BL/6J mice were obtained from The Jackson Laboratory. The Par4 ${ }^{-/-}$mice in a C57BL/6J background have been previously described (18) and characterized in the laser injury model (19). The Beth Israel Deaconess Medical Center Institutional Animal Care and Use Committee approved all animal care and experimental procedures.

Antibodies and reagents. Rat anti-mouse CD41 antibody (clone MWReg30) was from Emfret. Mouse anti-PDI monoclonal antibody
RL90, an IgG2a, from Affinity BioReagents, and a mouse anti-human fibrin II $\beta$-chain monoclonal antibody (clone NYBT2G1) from Accurate Chemical were purified by affinity chromatography using Protein A/G. Mouse IgG2a and IgG1 were obtained from BioLegend. Fab fragments of the anti-CD41 antibody were generated using the ImmunoPure Fab Preparation Kit from Pierce Biotechnology. Bacitracin A (Vetranal) was obtained from Sigma-Aldrich as protease-free. This material showed no protease activity. Sigma-Aldrich bacitracin was further purified by HPLC as instructed by the manufacturer. Rabbit polyclonal anti-bovine PDI antibody that cross-reacts with human and mouse PDI was obtained from Sigma-Aldrich and, for some experiments, affinity purified using bovine PDI covalently linked to cyanogen bromide-activated agarose. Fab fragments of anti-CD41 antibody, mouse anti-fibrin antibody, polyclonal anti-PDI antibodies, and monoclonal RL90 antibody as well as mouse IgG2a or polyclonal nonimmune IgG antibodies were labeled with Alexa Fluor 488 or Alexa Fluor 647 according to the manufacturer's instructions (Invitrogen). The molar ratio of Alexa Fluor to protein, determined spectrophotometrically, varied from 2.0 to 3.5 . 


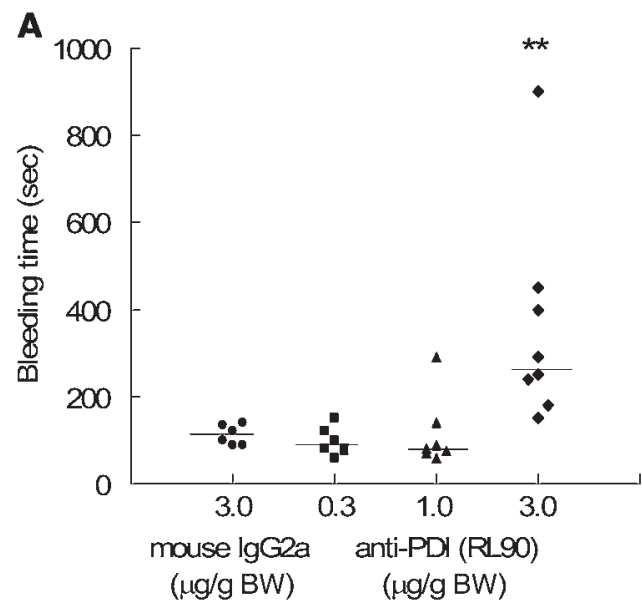

Figure 7

Effect of PDI antibody on tail bleeding time. (A) Tail bleeding times were determined as described in Methods following infusion of $0.3,1.0$, or $3 \mu \mathrm{g} / \mathrm{g}$ BW RL90 or $3 \mu \mathrm{g} / \mathrm{g} \mathrm{BW}$ isotype-matched IgG2a control antibody in 7, 7, 8, and 6 mice, respectively. Data are expressed as the time to the cessation of blood flow for greater than $10 \mathrm{~s}$; horizontal bars represent median tail bleeding time. The experiment was terminated at $15 \mathrm{~min}$ after tail cutting. (B) Blood loss during the bleeding time experiments was assayed by measuring the absorbance at $575 \mathrm{~nm}$ of hemoglobin $(\mathrm{Hb})$ in phosphate-buffered saline in which tails were immersed. Horizontal bars represent the median of bleeding times based on the absorbance of hemoglobin for each group of animals. ${ }^{\star *} P<0.01$ versus control, Dunn test after ANOVA (nonparametric).

Preparation of platelet lysates. Human platelet-rich plasma was prepared by centrifugation of sodium citrate-treated human blood at $200 \mathrm{~g}$ for $20 \mathrm{~min}$. The supernatant was collected and centrifuged at $700 \mathrm{~g}$ for $10 \mathrm{~min}$ in the presence of $0.5 \mu \mathrm{M}$ prostaglandin $\mathrm{E} 1\left(\mathrm{PGE}_{1}\right), 500 \mathrm{ng} / \mathrm{ml}$ prostacyclin $\left(\mathrm{PGI}_{2}\right)$, and $2 \mathrm{U} / \mathrm{ml}$ apyrase. The pellet was resuspended with HEPES-Tyrode buffer containing $10 \%$ sodium citrate solution, $0.25 \mu \mathrm{M} \mathrm{PGE}_{1}, 250 \mathrm{ng} / \mathrm{ml} \mathrm{PGI}_{2}$, and $1 \mathrm{U} / \mathrm{ml}$ apyrase and centrifuged at $800 \mathrm{~g}$ for $5 \mathrm{~min}$. For mouse platelet-rich plasma, sodium citrate-treated mouse blood obtained from 6- to 7 -week-old C57BL/6 mice was centrifuged at $150 \mathrm{~g}$ for $5 \mathrm{~min}$. The plasma fraction was recentrifuged at $150 \mathrm{~g}$ for $2 \mathrm{~min}$, and the supernatant was collected and centrifuged at $600 \mathrm{~g}$ for $5 \mathrm{~min}$ in the presence of $0.5 \mu \mathrm{M} \mathrm{PGE}_{1}$ and $500 \mathrm{ng} / \mathrm{ml} \mathrm{PGI}_{2}$. The pellet was suspended in HEPES-Tyrode buffer containing $10 \%$ sodium citrate solution, $0.25 \mu \mathrm{M} \mathrm{PGE}_{1}$, and $250 \mathrm{ng} / \mathrm{ml} \mathrm{PGI}_{2}$ and centrifuged at $600 \mathrm{~g}$ for $3 \mathrm{~min}$. Platelets were resuspended in HEPESTyrode buffer containing $2 \mathrm{mM} \mathrm{CaCl}_{2}$.

Specificity of PDI antibodies for buman and mouse PDI. Washed human $\left(1 \times 10^{8}\right.$ platelets $/ 100 \mu \mathrm{l})$ and mouse $\left(1 \times 10^{9}\right.$ platelets $\left./ 100 \mu \mathrm{l}\right)$ platelets were pelleted and solubilized in $100 \mu \mathrm{l}$ SDS lysis buffer ( $4 \%$ SDS and $6 \mathrm{M}$ urea containing $2 \mathrm{mM}$ EDTA and a protease inhibitor cocktail). The supernatant was electrophoresed under reduced conditions and immunoblotted with a rabbit polyclonal anti-bovine PDI antibody or a mouse monoclonal RL90 antibody. For experiments requiring releasate from activated platelets, mouse platelets $\left(1 \times 10^{9}\right.$ platelets $\left./ \mathrm{ml}\right)$ were incubated with $0.5 \mathrm{U} / \mathrm{ml}$ human thrombin for $5 \mathrm{~min}$ at $37^{\circ} \mathrm{C}$. The supernatant was collected by centrifugation at $1,000 \mathrm{~g}$ for $5 \mathrm{~min}$ and then subjected to additional centrifugation at $5,000 \mathrm{~g}$ for $5 \mathrm{~min}$ to remove any residual platelets. The supernatant was immediately used for the PDI activity assay and immunoblotting.

Assay of mouse PDI activity. Mouse PDI activity was monitored using the insulin transhydrogenase assay (29) and the releasate of thrombin-activated mouse platelets as a source of mouse PDI. Bovine insulin $(1 \mathrm{mg} / \mathrm{ml})$ in $250 \mu \mathrm{l}$ of $50 \mathrm{mM}$ Tris- $\mathrm{HCl}$ buffer, $\mathrm{pH} 7.5$, was incubated with $50 \mu \mathrm{l}$ mouse platelet releasate in the presence or absence of the monoclonal RL90 antibody or an isotype-matched IgG2a control, the IgG fraction of the rabbit polyclonal anti-bovine PDI antibody or the tered through a cannulus placed in the jugular vein. After the scrotum was incised, the testicle and surrounding cremaster muscle were exteriorized onto an intravital microscopy tray. The cremaster preparation was superfused with thermo-controlled $\left(36^{\circ} \mathrm{C}\right)$ and aerated $\left(95 \% \mathrm{~N}_{2}, 5 \% \mathrm{CO}_{2}\right)$ bicarbonate-buffered saline throughout the experiment. Microvessel data were obtained using an Olympus AX microscope with a $\times 600.9$ NA waterimmersion objective. The intravital fluorescence microscopy system has previously been described in detail (31). Digital images were captured with a Cooke Sensicam CCD camera in $640 \times 480$ format.

Laser-induced injury. Vessel wall injury was induced with a Micropoint Laser System (Photonics Instruments) focused through the microscope objective, parfocal with the focal plane and aimed at the vessel wall (14). Typically 1 or 2 pulses were required to induce vessel wall injury. The magnitude of injury as performed was comparable to the severe laser injury models described by others $(32,33)$, but distinct from the superficial or moderate injuries of the same groups. Multiple thrombi were studied in a single mouse, with new thrombi formed upstream of earlier thrombi to avoid any contribution from thrombi generated earlier in the animal under study. There were no characteristic trends in thrombus size or thrombus composition in sequential thrombi generated in a single mouse during an experiment. Image analysis was performed using Slidebook (Intelligent Imaging Innovations). Fluorescence data were captured digitally at up to 50 frames/s and analyzed as previously described (21). Typically, wide-field fluorescence images were captured at exposure times of $20 \mathrm{~ms}$, whereas bright-field images were captured with exposure times of $10 \mathrm{~ms}$. Data were collected for 3-5 min following vessel wall injury. The representative intravital color images were binarized using a thresholding procedure, in which the background is defined as the mean fluorescence of a mask upstream of the thrombus. The complete data sets of the representative and multiple identical experiments are presented graphically, plotting the integrated fluorescence intensity of all pixels in the image, regardless of their intensity, as a function of time. The kinetics of thrombus formation were analyzed by determining median fluorescence values over time in approximately $20-30$ thrombi (31). 
Tail bleeding times. Bleeding times were determined as previously described (18). Male mice (C57BL/6, 6-8 weeks old) were anesthetized, and mouse IgG2a or monoclonal RL90 anti-PDI antibody was injected through the jugular cannula. After $5 \mathrm{~min}$, the mouse tail was transected $5 \mathrm{~mm}$ from the tip with a razor blade. The bleeding tail was immersed in a $12-\mathrm{ml}$ test tube containing phosphate-buffered saline prewarmed at $37^{\circ} \mathrm{C}$, and time to cessation of blood flow was measured. Bleeding times were determined when the bleeding stopped for $10 \mathrm{~s}$. Bleeding times exceeding $15 \mathrm{~min}$ were noted as $15 \mathrm{~min}$. The amount of bleeding was quantitated by measuring the hemoglobin content of blood collected into $12 \mathrm{ml}$ of phosphate-buffered saline. Following centrifugation to pellet the red blood cells, the pellet was lysed with $5 \mathrm{ml}$ lysis buffer $\left(8.3 \mathrm{~g} / 1 \mathrm{NH}_{4} \mathrm{Cl}, 1.0 \mathrm{~g} / 1 \mathrm{KHCO}_{3}\right.$, and $0.037 \mathrm{~g} / \mathrm{l}$ EDTA), and the absorbance of the sample was measured at $575 \mathrm{~nm}$.

Statistics. For bleeding time experiments, data were statistically analyzed by ANOVA (nonparametric) using GraphPad Prism (GraphPad Software). If ANOVA conveyed a $P$ value less than 0.05 , a Dunn test was

performed to further assess significance. Differences were considered significant at $P \leq 0.05$.

\section{Acknowledgments}

We thank Vivien Chen for helpful discussions, Margaret Jacobs for assistance with the HPLC purification of bacitracin, and Snezna Rogelj for the gift of protease-free bacitracin. This work was supported by grants from the National Institutes of Health.

Received for publication October 3, 2007, and accepted in revised form January 30, 2008.

Address correspondence to: Bruce Furie, Beth Israel Deaconess Medical Center, 330 Brookline Avenue, Boston, Massachusetts 02215, USA. Phone: (617) 754-1200; Fax: (617) 754-1234; E-mail: bfurie@bidmc.harvard.edu.

1. Chen, V.M., and Hogg, P.J. 2006. Allosteric disulfide bonds in thrombosis and thrombolysis. J. Thromb. Haemost. 4:2533-2541.

2. Chen, K., Lin, Y., and Detwiler, T.C. 1992. Protein disulfide isomerase activity is released by activated platelets. Blood. 79:2226-2228.

3. Essex, D.W., Chen, K., and Swiatkowska, M. 1995. Localization of protein disulfide isomerase to the external surface of the platelet plasma membrane. Blood. 86:2168-2173.

4. Thomas, G., Skrinska, V.A., and Lucas, F.V. 1986. The influence of glutathione and other thiols on human platelet aggregation. Thromb. Res. 44:859-866.

5. Essex, D.W., and Li, M. 2003. Redox control of platelet aggregation. Biochemistry. 42:129-136.

6. Lahav, J., et al. 2002. Sustained integrin ligation involves extracellular free sulfhydryls and enzymatically catalyzed disulfide exchange. Blood. 100:2472-2478.

7. Lahav, J., et al. 2003. Enzymatically catalyzed disulfide exchange is required for platelet adhesion to collagen via integrin alpha2beta1. Blood. 102:2085-2092.

8. Burgess, J.K., et al. 2000. Physical proximity and functional association of glycoprotein 1 balpha and protein-disulfide isomerase on the platelet plasma membrane. J. Biol. Chem. 275:9758-9766.

9. Ahamed,J., et al. 2006. Disulfide isomerization switches tissue factor from coagulation to cell signaling. Proc. Natl. Acad. Sci. U. S. A. 103:13932-13937.

10. Versteeg, H.H., and Ruf, W. 2007. Tissue factor coagulant function is enhanced by protein-disulfide isomerase independent of oxidoreductase activity. J. Biol. Chem. 282:25416-25424.

11. Hogg, P.J. 2003. Disulfide bonds as switches for protein function. Trends Biochem. Sci. 28:210-214.

12. Wouters, M.A., Lau, K.K., and Hogg, P.J. 2004. Cross-strand disulphides in cell entry proteins: poised to act. Bioessays. 26:73-79.

13. Schmidt, B., Ho, L., and Hogg, P.J. 2006. Allosteric disulfide bonds. Biochemistry. 45:7429-7433.

14. Dubois, C., Panicot-Dubois, L., Merrill-Skoloff, G., Furie, B., and Furie, B.C. 2006. Glycoprotein VIdependent and -independent pathways of thrombus formation in vivo. Blood. 107:3902-3906.

15. Dubois, C., Panicot-Dubois, L., Gainor, J.F., Furie, B.C., and Furie, B. 2007. Thrombin-initiated platelet activation in vivo is $\mathrm{VWF}$ independent during thrombus formation in a laser injury model. J. Clin. Invest. 117:953-960.

16. Chen, V.M., et al. 2006. Evidence for activation of tissue factor by an allosteric disulfide bond. Biochemistry 45:12020-12028.

17. Ahamed, J., et al. 2007. Regulation of macrophage procoagulant responses by the tissue factor cytoplasmic domain in endotoxemia. Blood. 109:5251-5259.

18. Sambrano, G.R., Weiss, E.J., Zheng, Y.W., Huang, W., and Coughlin, S.R. 2001. Role of thrombin signalling in platelets in haemostasis and thrombosis. Nature. 413:74-78.

19. Vandendries, E.R., Hamilton, J.R., Coughlin, S.R., Furie, B., and Furie, B.C. 2007. Par4 is required for platelet thrombus propagation but not fibrin generation in a mouse model of thrombosis. Proc. Natl. Acad. Sci. U. S. A. 104:288-292.

20. Chen, K., Detwiler, T.C., and Essex, D.W. 1995 Characterization of protein disulphide isomerase released from activated platelets. Br. J. Haematol. 90:425-431.

21. Falati, S., Gross, P., Merrill-Skoloff, G., Furie, B.C., and Furie, B. 2002. Real-time in vivo imaging of platelets, tissue factor and fibrin during arterial thrombus formation in the mouse. Nat. Med. 8:1175-1181.

22. Bach, R., Konigsberg, W.H., and Nemerson, Y. 1988. Human tissue factor contains thioester-linked palmitate and stearate on the cytoplasmic half-cystine.
Biochemistry. 27:4227-4231.

23. Pendurthi, U.R., Ghosh, S., Mandal, S.K., and Rao, L.V. 2007. Tissue factor activation: is disulfide bond switching a regulatory mechanism? Blood. 110:3900-3908.

24. Essex, D.W.,Li, M., Miller,A., and Feinman, R.D. 2001. Protein disulfide isomerase and sulfhydryl-dependent pathways in platelet activation. Biochemistry. 40:6070-6075.

25. Jordan, P.A., et al. 2005. A role for the thiol isomerase protein ERP5 in platelet function. Blood. 105:1500-1507.

26. Yan, B., and Smith,J.W. 2001. Mechanism of integrin activation by disulfide bond reduction. Biochemistry. 40:8861-8867.

27. Yan, B., and Smith,J.W. 2000. A redox site involved in integrin activation. J. Biol. Chem. 275:39964-39972.

28. Butta, N., et al. 2003. Disruption of the beta3 663687 disulfide bridge confers constitutive activity to beta3 integrins. Blood. 102:2491-2497.

29. Holmgren, A. 1979. Thioredoxin catalyzes the reduction of insulin disulfides by dithiothreitol and dihydrolipoamide. J. Biol. Chem. 254:9627-9632.

30. Falati, S., et al. 2003. Accumulation of tissue factor into developing thrombi in vivo is dependent upon microparticle P-selectin glycoprotein ligand 1 and platelet P-selectin. J. Exp. Med. 197:1585-1598.

31. Dubois, C., Atkinson, B., Furie, B.C., and Furie, B. 2006. Real time in vivo imaging of platelets during thrombus formation. In Platelets. A.D. Michelson, editor. Academic Press/Elsevier. Amsterdam, The Netherlands. 611-626.

32. Nonne, C., et al. 2005. Importance of platelet phospholipase Cgamma2 signaling in arterial thrombosis as a function of lesion severity. Arterioscler. Thromb. Vasc. Biol. 25:1293-1298.

33. Mangin, P., et al. 2006. Thrombin overcomes the thrombosis defect associated with platelet GPVI/ FcRgamma deficiency. Blood. 107:4346-4353. 